$\xi=\mathrm{\alpha}$

\title{
Experimental analysis and comparison of tropospheric scintillation prediction models using eutelsat-36b satellite in a tropical Nigeria
}

\author{
Joseph Sunday Ojo ${ }^{1 *}$, Babatunde Rabiu ${ }^{2}$, Sandro M Radicella ${ }^{3}$, Obiseye O. Obiyemi ${ }^{3}$ \\ ${ }^{1}$ Department of Physics, Federal University of Technology Akure, Nigeria \\ ${ }^{2}$ Centre for Atmospheric Research, National Space Research and Development Agency, \\ Federal Ministry of Science and Technology, Anyigba, Nigeria \\ ${ }^{3}$ Telecommunications/ICT for Development Laboratory, The Abdus Salam International Centre, for Theoretical Physics, Trieste, Italy \\ ${ }^{4}$ Department of Electrical and Electronics, Osun State University, Osogbo, Nigeria \\ *Corresponding author E-mail: josnno@yahoo.com
}

\begin{abstract}
Knowledge on clear-air effects is of paramount importance to proper link budgeting for optimum communication systems design performances. In this paper, one-year (January - December 2013) tropospheric scintillation data are extracted from the EUTELSAT-36B Kuband satellite measurements installed at Akure (Lat: $7.17^{\circ} \mathrm{N}$, Long: $5.18^{\circ} \mathrm{E}$, Alt: $358 \mathrm{~m}$ ) for statistical analysis and the result compared with some established troposphere scintillation models in order to obtain the best prediction model performance for this region. The result shows that even in the absence of rain, tropospheric scintillation shows a strong seasonal effect in this region up to amplitude above $0.92 \mathrm{~dB}$. The scintillation intensity fits better to gamma distribution at a high scintillation level taken into consideration the local meteorological parameters. Models comparison with experimental data also shows that the Karasawa model with the lowest percentage error of about $7 \%$ was found to be best fit for predicting propagation impairment relating to be fading at a Ku band frequency in this region. The overall results will provide information on scintillation margin needed for sizing antennas and amplifiers for reliable performance and the average bit-error probability on a scintillation-degraded digital satellite link in this region.
\end{abstract}

Keywords: Tropospheric Scintillations; Trodan Data; Tropical Climate; Satellite Communication.

\section{Introduction}

The fast growth of technology in the present day cannot be overemphasized. New technological systems are being developed every day to enhance better performance. This includes, but not limited to the introduction of mobile and fixed digital telecommunications in the K-band (Ku and Ka-band Frequencies) based on very small aperture terminals (VSAT), clusters of low-elevation orbit (LEO) satellites and wireless multipoint distribution services (MDS) with link margins for high availability [1-3]. In order to meet up with the challenges, there is a need to adapt some features into the system designs among which involved increase of channel frequency and the decrease of the antenna aperture and elevation angle. Aside rain attenuation effect on signal operating at high frequencies (> $10 \mathrm{GHz}$ ), tropospheric scintillations have been described as one of the major problems in the link budget design of microwave and millimeter-wave communication systems [4-6]. It results in a significant degradation of the signal-to-noise ratio (up to several decibels) and their effects increase as the operating frequency increases and the look-angle elevation of the satellite decreases.

Furthermore, troposphere scintillation is known to show a strong correlation with some meteorological parameters such as temperature, humidity and refractive index variations of propagation media [7]. High temperature and humidity give the greatest scintillation amplitudes for a given path. Tropical climates are character- ized by high temperature and high humidity; hence they have higher scintillation amplitude than temperate climates. Troposphere scintillation is generated by the small-scale fluctuations of the refractive index due to turbulence, which affects the curvature of the electromagnetic wave path and gives some insight into the fading phenomenon and enhancements of the received signal amplitude [8]. Generally speaking, a more significant concern is that scintillation occurs continually, irrespective of the clearness of the sky, because the temperature and humidity remained in the air even on bright days. Therefore, to achieve systems with low fade margin, scintillation effects must be properly accounted during link budgeting, especially those at high frequency and low elevation angles [9].

In a similar work carried out by Mandeep and Islam [6] on the subject matter, one-year data (1999-2000) measured using SUPERBIRD-C satellite in Tronoh, Malaysia was used to estimate and compared with some of the statistical troposphere models. Eight of the statistical models of monthly mean scintillation intensity were reviewed, and their predictions compared with measurements. Based on their results, the Karasawa and Ortgies-T models were found to have the best overall performance in the region. Cheng and Mandeep [10] also analyzed tropospheric.

Scintillation based on data from a satellite link installed in Bandung, Indonesia, at a frequency of $12.247 \mathrm{GHz}$ from 1999 to 2000. The data are processed and compared with the predictions of several well-known scintillation prediction models. The result revealed that the ITU-R model gave the lowest error rate when pre- 
dicting the scintillation intensity for fade at $4.68 \%$. These two results, among others [3 -5] indicated that there is no universal model, especially in the tropical regions due to the variation of the meteorological parameters. Hence, generalizing signal degradation arising from the clear-air effect may either underestimate or overestimate the link budgeting needed in the design of satellite communication systems. Although this paper used the method earlier adopted by [6] and [10], the differences lie in the usage of the entirely new measurement site with different meteorological parameters, different years of measurements, different satellite look angle and the type of rain gauge used.

The subject matter has also started receiving attention in Nigeria. For example, in the work carried out by Agunlejika et al. [11] on the subject matter, scintillation prediction over some randomly selected cities was estimated based only on ITU model. However, the result was never tested with any real-time experimental measurement.

In this study, we have estimated and compare the level of troposphere scintillation that could be encountered through the Earthspace links in a tropical climate based on characterization and analysis of time series EUTELSAT-36B Ku-band satellite signal and make a comparison with the existing scintillation prediction models. The models are used as an interaction tool between microwave radiations and turbulent medium to evaluate the received scintillation power and its spectrum in a given frequency band and for a given elevation angle [12] and to also propose which of them will fit the region under consideration. The troposphere scintillation models are deduced based on local meteorological data namely: temperature $(\mathrm{t})$, pressure $(\mathrm{P})$ and relative humidity $(\mathrm{H})$ obtained from the databank of the Tropospheric Data Acquisition Network (TRODAN) ground observation measurement.

The remaining part of this paper is organized as follows. Section 2 gives the overview of some scintillation prediction models. This is followed by information on experimental setup and data processing in section 3. Section 4 provides detailed results and discussion. Finally, Section 5 concluded.

\section{Overview of some scintillation prediction models}

A detailed estimation of the accessibility of a satellite connection can be obtained based on the knowledge about the distribution of scintillation intensities, which expresses the level of scintillation amplitude fluctuation. There exist several models for predicting troposphere scintillation based on meteorological measurement Among the numerous models, we have selected four prediction models for this study: Otung [13], Van de Kamp [14], Karasawa [15], and ITU-R P. 618-12 [16]. The models were selected based on their correlation with the wet refractive index value and the ability to predict long-term distribution propagation signals. The major parameters used by most of these models are as follows: $\sigma_{\text {ref }}$ is the standard deviation of the signal amplitude. $\mathrm{G}(\mathrm{x}$ is an antenna averaging factor, $\theta$ represents the path elevation angle. $F$ is the operational frequency. Nwet represents the wet term of the troposphere refractivity $\left(3.73 \times 10^{5} \mathrm{e} / \mathrm{T}^{2}\right)$, e and $\mathrm{T}$ are the water vapor pressure $(\mathrm{mb})$ and absolute ambient temperature averaged over the period (> one month), D is the diameter of the earth-station antenna $(m), \eta$ It represents the antenna efficiency, and $p$ is the percentage of time (\%)- all these parameters are readily available. Each model gives the value of the scintillation parameter exceeded on a given satellite link during $\mathrm{p} \%$ of the period.

\subsection{ITU-R model}

The ITU-R scintillation model based on measurements and theoretical frequency dependence and aperture averaging effects, estimates the average scintillation intensity in terms of the standard deviation of the deviation of signal fluctuation due to scintillation, $\sigma$ asi over a minimum period than one month. The model is represented as: $\sigma_{a i}=\frac{\sigma_{r i f} f^{7 / 12} g(x)}{(\sin \theta)^{1.2}}$

Where

$\sigma_{n f}=3.6 \times 10^{-3}+1.03 \times 10^{-4} N_{w e}$

$g(x)=\left\{3.86\left(x^{2}+1\right)^{11 / 2} \sin \left[\frac{11}{6} \arctan (1 / x)\right]-7.08 x^{5 / 6}\right\}^{1 / 2}$

$x=0.058 k(D \eta)^{2} / L$

$L=2 h / \sqrt{\sin ^{2}(\theta)+(2 h / \operatorname{Re})}+\sin (\theta)$

Other parameters are $\mathrm{h}=1000 \mathrm{~m}$ is the height of the turbulent, $\mathrm{Re}$ $\sim 8.5 \times 10^{6} \mathrm{~m}$ is the effective Earth radius and $\mathrm{k}$ are the wave number $\left(\mathrm{m}^{-1}\right)$.

Hence, scintillation fading A (p) can be obtained for $0.01<\mathrm{p}<50$ as:

$$
A(p)=\sigma_{a s i}\left(\begin{array}{l}
-0.061\left(\log _{10} p\right)^{3}+0.072\left(\log _{10} P\right)^{2} \\
-1.7\left(\log _{10} p\right)+3.0
\end{array}\right)
$$

\subsection{Otung model}

This model is based on the modification of ITU-R Model by considering the theoretical dependence of $\sigma$ asi on $\theta$ as $\sin (\theta)^{-11 / 12}$. Thus transforming equation (1) as:

$\sigma_{a i}=\frac{\sigma_{r f} f^{7 / 12} g(x)}{(\sin \theta)^{-\frac{11}{12}}}$

Thus, scintillation fading A (p) and enhancement E (p) for signal level can be obtained respectively as:

$$
\begin{aligned}
& A(p)=3.6 \sigma_{\text {axi }} \exp \left(-\frac{9.5 \times 10^{-4}}{p}-[0.4+0.002 p] \ln (p)\right) \\
& E(p)=3.17 \sigma_{\text {axi }} \exp \left(-9.5 \times 10^{-4} p-[0.272+0.004 p] \ln (p)\right)
\end{aligned}
$$

\subsection{Van de kamp model}

The Van de Kamp slightly modifies the ITU-R Model as represented by:

$\sigma_{a i}=\sigma_{r f} f^{0.15} \frac{g(x)}{(\sin \theta)^{1.3}}$

Also, scintillation fading A (p) and enhancement E (p) for signal level can be obtained respectively as:

$$
\begin{aligned}
& A(p)=a_{1}(p) \sigma_{a x i}+a_{2}(p) \sigma_{a i}^{2} \\
& E(p)=a_{1}(p) \sigma_{a i}+a_{2}(p) \sigma_{a i}^{2}
\end{aligned}
$$

Where

$$
\begin{aligned}
& a_{1}(p)=-0.0515(\log p)^{3}+0.206(\log p)^{2}-1.81(\log p)+2.81 \\
& a_{2}(p)=-0.172(\log p)^{2}-0.454(\log p)+0.274
\end{aligned}
$$




\subsection{Karasawa model}

The Karasawa model for the predicted scintillation intensity is given as:

$$
\sigma_{a s i}=\frac{\sigma_{n} f^{0.45} \sqrt{G\left(D_{a}\right)}}{\sin (\theta)^{1.3}} \quad \text { For } \theta>5^{\circ}
$$

Where $\sigma_{n}$ is the normalized intensity and $G\left(D_{a}\right)$ is the antenna aperture averaging factor given by:

$$
G\left(D_{a}\right)= \begin{cases}1.0-1.4 \frac{D_{a}}{2 \sqrt{\lambda L}} & ; 0 \leq \frac{D_{a}}{2 \sqrt{\lambda L_{d}}} \leq 0.5 \\ 0.5-0.4 \frac{D_{a}}{2 \sqrt{\lambda L}} & ; 0.5 \leq \frac{D_{a}}{2 \sqrt{\lambda L_{d}}} \leq 1.0 \\ 0.1 & ; 0 \leq \frac{D_{a}}{2 \sqrt{\lambda L_{d}}} \leq 0.5\end{cases}
$$

And

$$
\sigma_{n}=3.42 \times 10^{-3}+1.1856 \times 10^{-4} N_{w e t}
$$

Where $\lambda$ is the wavelength in $m, D_{a}$ is the effective antenna diameter and $\mathrm{L}_{\mathrm{d}}$ is the distance of the turbulent part of the path given as:

$$
L_{d}=2 \frac{h}{\sqrt{\sin ^{2} \theta+2 \frac{h}{\operatorname{Re}}+\sin \theta}}
$$

The corresponding signal fading is given as:

$$
A(p)=\sigma_{a s i}\left(\begin{array}{l}
-0.06\left(\log _{10} p\right)^{3}+0.08\left(\log _{10} P\right)^{2} \\
-1.25\left(\log _{10} p\right)+2.67
\end{array}\right) \text { For } 0.01<\mathrm{p}<50
$$

While the signal enhancement can be expressed as:

$$
E(p)=\sigma_{a s i}\left(\begin{array}{l}
-0.06\left(\log _{10} p\right)^{3}+0.072\left(\log _{10} P\right)^{2} \\
-1.7\left(\log _{10} p\right)+3.0
\end{array}\right)
$$

\section{Experimental set-up and data processing}

The experimental site is at the Federal University of Technology, Akure FUTA (Lat: $7.17^{\circ} \mathrm{N}$, Long: $5.18 \mathrm{oE}$, Alt: $358 \mathrm{~m}$ ), Nigeria. The meteorological data measured from January 2012 to December 2013 was obtained from the databank of the Tropoxspheric Data Acquisition Network (TRODAN) propagation measurement. TRODAN formerly known as Nigeria Environmental and Climatic Observatory Program (NECOP) is a project that was set up to monitor the lower atmosphere. The lower atmosphere is a region which covers from the surface of the Earth to the altitude of about $11 \mathrm{~km}$. This project is designed to collect and provide real-time meteorological data from different locations across Nigeria using automatic weather stations for the purpose of research and development [17]. The automatic weather stations comprise of sensors in unit that measured soil moisture content (volume of water), wind speed and wind direction, air and soil temperature, relative humidity, rain rate among others. Detailed description about the TRODAN set-up is available in [17], [18]. The meteorological variables needed in this work are the water vapour pressure $(\mathrm{mb})$ and absolute ambient temperature averaged over the period of each month for the determination of the wet term of the troposphere refractivity. Manual sorting was carried out on raining events based on $0.2 \mathrm{~mm}$ tipping bucket rain gauge which is time synchronized measurement [8].
The beacon experimental data were extracted from a $12.245 \mathrm{GHz}$ Ku-band signal EUTELSAT 36B measurements installed at an elevation angle of $42^{\circ}$ and at a sampling rate of $1 \mathrm{~Hz}$. A small integration time of $1 \mathrm{~Hz}$ is chosen to sufficiently characterize troposphere scintillation as reported in the work of Cheng and Mandeep [10]. The $0.6 \mathrm{~m}$ offsetted parabolic dish antenna of the beacon measurement was fixed upon a pole in a fenced area besides Physics building of the University. Sampling of the received signal at every $0.1 \mathrm{~s}$ was done using the spectrum analyser placed inside the Communication Research Laboratory (CRL). The whole measurement spans between January and December 2013 and comprises both the rainy and dry season months. During the observation period, the availability of data was about $96.8 \%$. The remaining $3.2 \%$ of unavailability of the data was due to system maintenance.

As reported in [15], scintillation usually occurs irrespective of whether the sky is clear or rainy. During rainy periods, the level at which signal fluctuates as a result of troposphere scintillation usually accompanied signal-level attenuation due to rain, non-rain events were separated from the rain events in the experimental data. In addition, any spurious samples resulting from loss of lock due to the satellite propellant saving option and satellite movement were visually removed from the raw data [6].

The analysis of the scintillation intensity reported in this paper is estimated based on the standard deviation of the amplitude fluctuations integrated over $1 \mathrm{~min}$. The beacon level is displayed in $\mathrm{dB}$ in a real time on the Sat link meter and subsequently stored in the hard disk. To achieve better spectral analysis, the data were extracted by passing through a fifth-order high-pass Butterworth filter with a $0.04-\mathrm{Hz}$ cut-off frequency. After the filtering process, the resulting data consists of positive (enhancement) and negative (fade) scintillation amplitude fluctuations above the mean level [19], [20]. Performance evaluation of each of the scintillation models is therefore tested based on the fractional percentage error.

\section{Analysis and results}

This section gives a comprehensive analysis of the distribution of scintillation intensity and relates it to peak to peak at scintillation amplitude fluctuations. Many useful findings about the probability distribution of intensity and the seasonal and diurnal pattern of scintillation are reported.

\subsection{Analysis and characterization of clear-sky scintilla- tion}

Analysis and characterization of the results of clear-sky scintillation is presented throughout this section.

\subsubsection{Time series analysis of clear-sky scintillation}

Figure 1 shows a typical signal level variation of troposphere scintillation by the broadcasting satellite at $12.245 \mathrm{GHz}$. The scintillation level of about $1.6 \mathrm{~dB}$ peak-to peak (pp) was obtained with a peak extracted variance of about $-0.15 \mathrm{~dB} 2$. The dynamic of the clear sky could be seen based on the $0 \mathrm{~dB}$ in the plot. The rapid fluctuation in the Earth-space signal tends to be enhanced by the atmospheric turbulent, especially the cloud liquid water and this could lead to scintillation. The fluctuation observed in figure 1 shows a clear influence of rain-induced attenuation in the troposphere scintillation time series. However, using the cut-off frequency of $40 \mathrm{mHz}$ as presented in figure. 2, the actual effect of scintillation with the absence of rain-induced attenuation could be seen. The results will assist in the designing an adaptive algorithm for power control, coding rate technique as well as other countermeasures for microcell diversity [13]. 


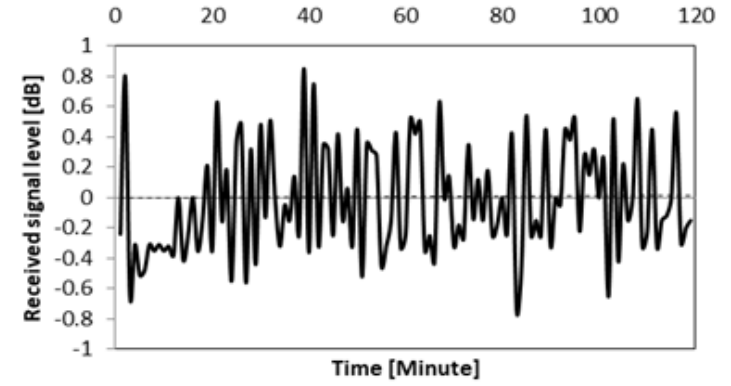

Fig. 1: Typical Example of Tropospheric Scintillation Observed with A Cutoff Frequency of 4-MHz for $120 \mathrm{Min}$.

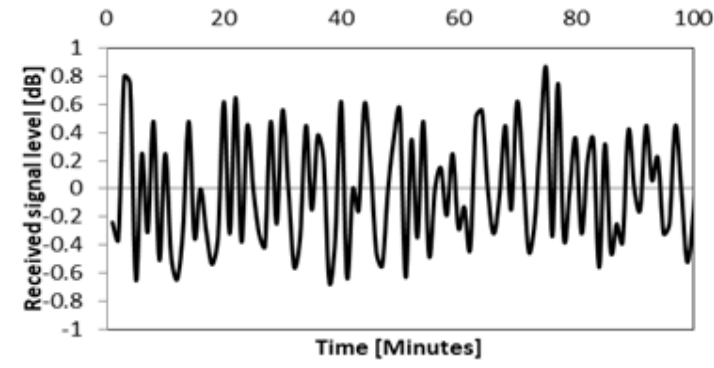

Fig. 2: Typical Example of Tropospheric Scintillation Observed with A Cutoff Frequency of 40-MHz for $100 \mathrm{Min}$.

\subsubsection{Probability distribution of tropospheric scintillation}

A probability density function presents how likely to observe a specific value at any given point for any random variable. It also gives the ability to predict the expected value of any process at any given time. Two probability distribution functions commonly used are considered throughout this work namely: the log-normal distribution and the gamma distribution models.

For the log-normal distribution, a positive random value as the scintillation amplitude $\chi$ is said to be log-normally distributed if the logarithm of $\chi$ is normally distributed [21],

$\ln (\chi) \sim \mathrm{N}\left(\mu, \sigma^{2}\right)$

If $\phi$ and $\eta$ represents the cumulative probability distribution function and the probability density function respectively for $\mathrm{N}(0.1)$ distribution then, the probability density distribution can be obtained follows:

$$
\begin{aligned}
& f \chi(x)=\frac{d}{d x} \operatorname{pr}(\chi \leq x)=\frac{d}{d x} \operatorname{pr}(\chi \leq x \ln ) \\
= & \frac{d}{d x} \phi\left(\frac{\ln x-m}{\sigma}\right) \\
= & \eta\left(\frac{\ln x-m}{\sigma}\right) \frac{d}{d x}\left(\frac{\ln x-m}{\sigma}\right) \\
= & \eta\left(\frac{\ln x-m}{\sigma}\right) \frac{1}{\sigma x} \\
= & \frac{1}{\sigma x \sqrt{2 \pi}} \exp \left[\frac{1}{2}\left(\frac{\ln x-m}{\sigma}\right)^{2}\right] x>0
\end{aligned}
$$

For the cumulative distribution function, the variable function is given as:

$$
F \chi(x)=\phi\left(\frac{\ln x-m}{\sigma}\right)
$$

Where $\phi$ is the cumulative distribution function for a standard normal distribution representing $\mathrm{N}(0.1)$. Equation (26) can also be expressed as:

$$
F \chi(x)=\left[1+e r f\left(\frac{\ln x-m}{\sigma \sqrt{2}}\right)\right]=\frac{1}{2} \operatorname{erfc}\left(\frac{\ln x-m}{\sigma \sqrt{2}}\right)
$$

Where erfc is the complementary error function.

Gamma distribution contains wide range of specific distributions with the probability density function for scintillation amplitude $\chi$ is given as:

$$
f \chi(x)=\frac{\beta^{\alpha}}{\Gamma(\alpha)} x^{\alpha-1} e^{-\beta x} \text { For } \mathrm{x}>0 \text { and } \alpha, \beta>0
$$

Where $\Gamma(\alpha)$ is a complete gamma function.

Also, the cumulative distribution function is expressed as;

$$
F \chi(x)=\frac{1}{\Gamma(\alpha)} \gamma(\alpha, \beta x)
$$

Where $\gamma(\alpha, \beta \mathrm{x})$ is the incomplete gamma function.

The parameters $\alpha$ and $\beta$ are related to standard deviation, mean and variance as:

$$
\beta=\frac{m}{\sigma^{2}} \text { And } \alpha=\left(\frac{m}{\sigma}\right)^{2}
$$

Where $\mathrm{m}$ and $\sigma$ are the measured mean and standard deviation of $\chi$ respectively.

Figure 3 presents the long-term probability density function (pdf) of scintillation amplitude series extracted using a high-pass filter cutoff frequency, fc of $0.04-\mathrm{Hz}$ for the period under review. Although both lognormal and gamma distributions show similar shapes, the gamma model proved to provide a good performance for the scintillation amplitude while the lognormal departs from the pdf-measured scintillation intensity. It could also be seen that the pdf has a positive skew for a long-term observational period, especially for the strongest intensities. This should be expected due to the absence of rain as a result of the cumulus clouds that lead to a burst of strong scintillation during the event of cumulus clouds across the path [10]. The information from the pdf scintillation amplitude may assist to derive the probability of average biterror on the scintillation-fading digital satellite link in the region [13], [22].

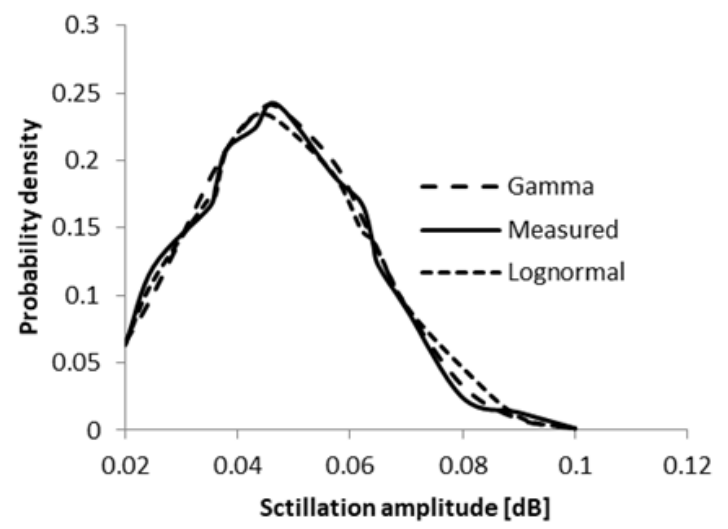

Fig. 3: Long -Term Probability Distribution of Scintillation Amplitude.

\subsubsection{Monthly and annual analysis of tropospheric scintilla- tion}

Figure 4 presents the monthly mean value of measured scintillation intensity, $\sigma_{\text {ref. }}$ It is estimated by calculating the standard deviation of the signal amplitude over an one-minute interval from the 
attenuation of time series. The mean value scintillation intensity is generally stronger in wet seasons than dry season. The fact remains that the mean monthly scintillation intensity depends on the wet term of the troposphere refractivity $\mathrm{N}_{\text {wet }}$-see equation (2) under section 2.1. The value reaches a maximum of $0.07 \mathrm{~dB}$ in October and followed by $0.065 \mathrm{~dB}$ in July.

The monthly maximum of measured scintillation intensity is also presented in figure 5. More usually, the average scintillation intensity has generally stronger influence in the dry season than wet season. The scintillation intensity reaches the maximum of $2.2 \mathrm{~dB}$ in November and following by $1.9 \mathrm{~dB}$ in January.

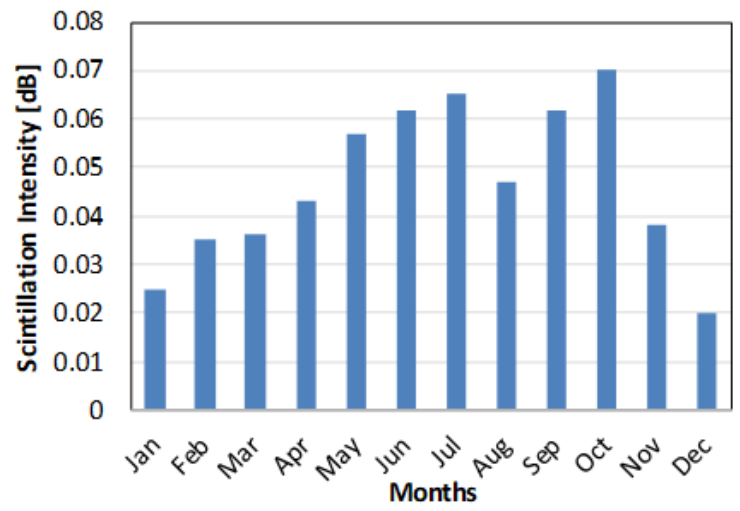

Fig. 4: Monthly Mean Values of Measured Scintillation Intensity.

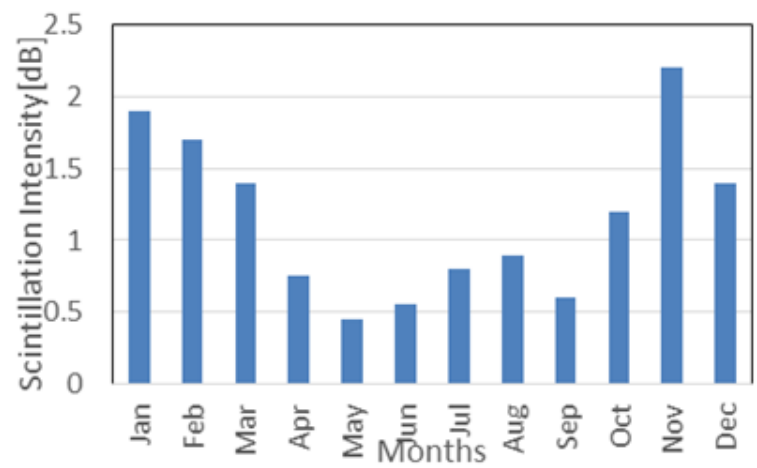

Fig. 5: Monthly Maximum Occurrence of Measured Scintillation Intensity.

The cumulative distribution functions (CDF) of the scintillation enhancement amplitude (positive log-amplitude) and fade amplitude (negative log-amplitude) is presented in figure.6. The longterm scintillation amplitude is processed only during clear-sky events. The negative signal deviations or fade amplitude is averagely larger than the positive log-amplitude enhancement because of the large signal fluctuations caused by the refractive index in homogeneities impacted by strong atmospheric turbulence in the clouds as the signal propagates through the troposphere. The fade signal level deviates from the enhancement level at $0.7 \%$ of time and the magnitude increases towards the lower time percentages. The deviation may be due the presence of the cloud liquid water along the propagation path, and the commencement of the formation of convective rain type [23]

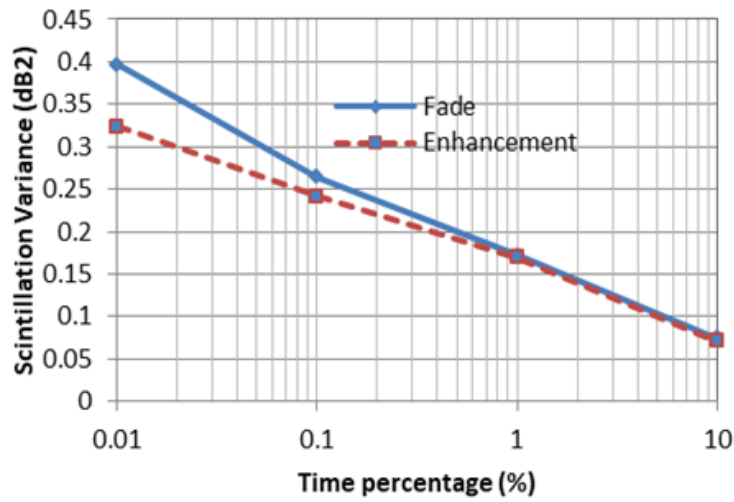

Fig. 6: Annual Cumulative Distribution of Signal Level.

\subsection{Performance of scintillation models}

Figure 7 ( $a$ and $b$ ) shows the comparison of the measured data with the predicted model for fade and enhancement level respectively. The comparison is to understand the limits of each prediction model and the degree of the applicability in this region. In figure 7 (a), the Karasawa model shows an approximate close agreement with the measured signal fading scintillation amplitude values for the entire prediction percentage time. This is because the model was developed based on the data collected during norain period with a strong relation between the scintillation characteristics, and the water vapour contribution effect on the refractivity index obtained from the local humidity and ground temperature data. The model was developed based on higher elevation angles and temperature. The ITU model follows after Karasawa model, while Otung model deviates significantly from other models inclusive of the measured scintillation fading in this region. This is due to the difference in the elevation angle, the frequency and the location of the experimental data that were collected.

In figure 7 (b) which illustrates the comparison with the signal enhancement scintillation measurements with the predicted models, the Karasawa, Van de Kamp and Otung models follow the same trend as fading with little deviation in their predicted values. Otung model still maintained the lowest deviation from the measured data. ITU model is omitted because the model was not designed to produce results in scintillation enhancement.

(A)

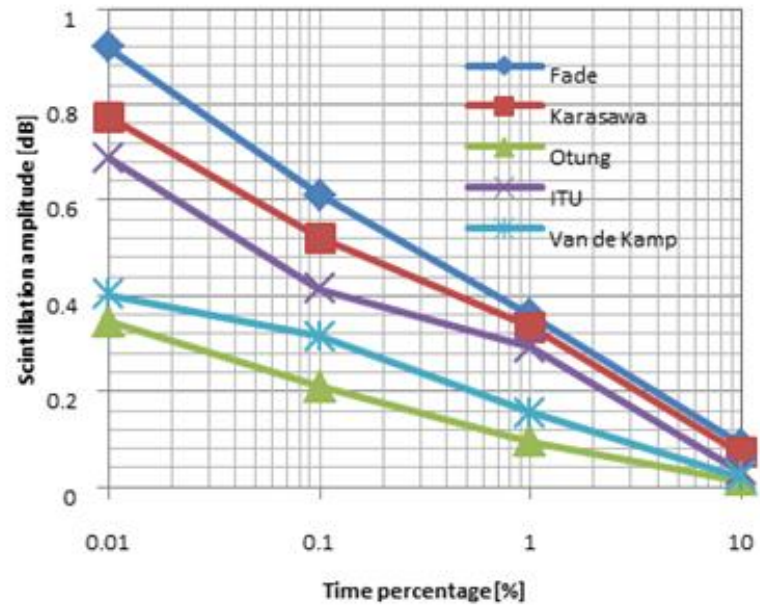


(B)

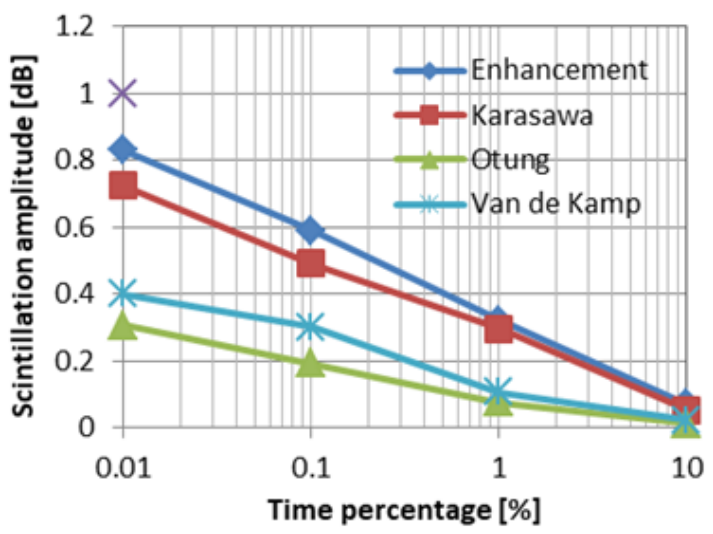

Fig. 7: Comparison of the Measured Data with the Predicted Model for (A) Fade and (B) Enhancement.

The comparison between the measured and the models can better be presented based on the statistical analysis using the percentage fractional error as:

$$
\varepsilon=\frac{A(p)_{\text {measurud }}-A(p) \text { predicted }}{A(p)_{\text {measured }}} 100 \%
$$

Where A (p) is the Scintillation amplitude at a specific time percentage, p. The same is applicable to enhancement, $\mathrm{E}(\mathrm{p})$ as the case may be.

Table 1 presents the statistical comparison between fades and enhancement with scintillation prediction models. We observed that at all the percentages of time considered; Karasawa model gave the lowest percentage error with minimum error of about $7 \%$ at $1 \%$ of the time (scintillation fading and enhancement) and maximum of $20 \%$ and $25 \%$ at $10 \%$ of the time for scintillation fading and enhancement respectively. It follows closely to the Karasawa model is the ITU model for scintillation fading where the percentage error range between 19 and $63 \%$ at $0.01-10 \%$ of the time. Al other models have a high error percentage with Otung model giving the highest error values for both fade and enhancement scintillation. Judging from this analysis and based on the present data, Karasawa model was found to be best fit for predicting propagation impairment relating to scintillation in the tropical region under consideration.

We also examined the seasonal variation of scintillation intensity as presented in figure 8 . We observed strong scintillation events between November and March in the measured data, the Karasawa and Otung model. However, the reverse is the case for the remaining two models (Van de Kamp and ITU), which show weak scintillation event during the same months. The strong scintillation events may be as a result of high temperatures and the presence of convective clouds during the period. As a matter of fact, during this period, the ground temperature can be as high as $32^{\circ} \mathrm{C}$, which is synonymous with the coverage of cumulus clouds across the sky [6]. Thus, it can be concluded that scintillation increases when the density of water vapour in the atmosphere is higher, which may be one of the reasons why it is more strongly revealed around the months of November and February, with its high temperatures and high humidity. However, the weak scintillation observed during same months by the ITU and Van de Kamp models may be as a result of the high dependence on the $\mathrm{N}_{\text {wet }}$ of the rain events. This may also be one of the reasons why ITU model shows strong scintillation during the wet seasons. Scintillation strength is therefore, stronger during the dry-season months when compared to the wet season months. Furthermore, it can be stated that the high scintillation strength is associated with high humidity and $\mathrm{N}_{\text {wet }}$ in dry season. The summary of the monthly variability of standard deviation of the scintillation intensity is presented in Table 2 .

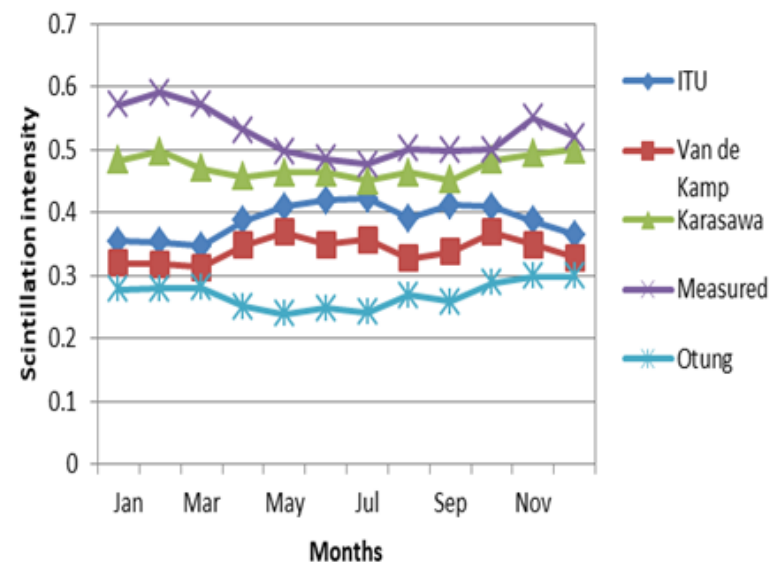

Fig. 8: Monthly Variability of Standard Deviation of the Scintillation Intensity Compared with Prediction Models.

\section{Conclusion}

Experimental analysis and comparison of clear-air scintillation prediction models have been presented in this paper. Four models, namely: Karasawa, ITU-R, Van de Kamp, Otung models were compared with the measured scintillation data obtained from the EUTELSAT-36B Ku-band en route over a tropical climate in Nigeria. The measured fades to stretch up to $0.92 \mathrm{~dB}$ at $0.01 \%$ of the time, while the measured enhancements stretch up to $0.83 \mathrm{~dB}$ at $0.01 \%$ of the time. Scintillation strength is also observed to be stronger during the dry season when compared to the wet season in this region. Based on the percentage fractional error statistical analysis, Karasawa model gave the lowest percentage error with minimum error of about $7 \%$ when compared with the measured scintillation data. This is closely followed by the ITU scintillation model. In overall, Karasawa model gave the best scintillation intensity predictions and found to be suitable for use in Akure and its environs.

Table 1: Percentage Fractional Error of the Models

\begin{tabular}{|c|c|c|c|c|c|c|c|}
\hline \multirow[t]{2}{*}{ Time Percentage } & \multicolumn{4}{|c|}{$\begin{array}{l}\text { Fractional Error \% } \\
\text { Fades }\end{array}$} & \multicolumn{3}{|c|}{ Enhancement } \\
\hline & Karasawa & ITU & Van de Kamp & Otung & Karasawa & Van de Kamp & Otung \\
\hline 0.01 & 15.83331 & 25.21377 & 56.15921 & 62.46375 & 12.73090 & 56.52032 & 66.81157 \\
\hline 0.1 & 14.56581 & 31.99118 & 48.38463 & 65.54942 & 16.75449 & 50.67971 & 68.82811 \\
\hline 1 & 7.094454 & 19.03890 & 57.05565 & 73.72232 & 7.981261 & 70.94454 & 79.27787 \\
\hline 10 & 20.17614 & 63.50948 & 75.73170 & 84.62059 & 25.94075 & 76.84281 & 86.84281 \\
\hline
\end{tabular}


Table 2: Monthly Variability of Standard Deviation of the Scintillation Intensity

\begin{tabular}{llllllllllllll}
\hline & Jan & Feb & Mar & Apr & May & Jun & Jul & Aug & Sep & Oct & Nov & Dec & Average \\
\hline ITU & 0.3545 & 0.3531 & 0.3471 & 0.3871 & 0.4095 & 0.4195 & 0.4215 & 0.3915 & 0.4115 & 0.4090 & 0.3874 & 0.3644 & 0.3880 \\
Van de Kamp & 0.3191 & 0.3179 & 0.3124 & 0.3484 & 0.3686 & 0.3486 & 0.3576 & 0.3276 & 0.3376 & 0.3682 & 0.3487 & 0.3280 & 0.3402 \\
Karasawa & 0.4838 & 0.4972 & 0.4701 & 0.4573 & 0.4638 & 0.4638 & 0.4504 & 0.4638 & 0.4518 & 0.4832 & 0.4938 & 0.5007 & 0.4733 \\
Measured & 0.5711 & 0.5911 & 0.5711 & 0.5309 & 0.4971 & 0.4852 & 0.4771 & 0.5011 & 0.4991 & 0.5005 & 0.5511 & 0.5213 & 0.5247 \\
Otung & 0.2771 & 0.2793 & 0.2801 & 0.2509 & 0.2381 & 0.2481 & 0.2418 & 0.2681 & 0.2581 & 0.2880 & 0.2981 & 0.2981 & 0.2688 \\
\hline
\end{tabular}

\section{Acknowledgement}

The results presented within this paper rely on TRODAN data collected and managed by the Centre for Atmospheric Research, National Space Research and Development Agency, Federal Ministry of Science and Technology, Anyigba, Nigeria. We thank the Centre for Atmospheric Research and their partners for promoting high standards of atmospheric observatory practice as well as the Federal Government of Nigeria for continuous funding of the Nigerian Space programme (www.carnasrda.com). Authors are also grateful to the International Centre for Theoretical Physics (ICTP), Trieste Italy for access to some of the materials used in this work.

\section{References}

[1] Rappaport, T. S, 2002, Wireless Communications, 2nd ed., New York: Prentice Hall.

[2] Matricciani. E. Mauri, M \& Riva C. (1996), Relationship between scintillation and rain attenuation at $19.77 \mathrm{GHz}$, Radio Sci., 31(2), 273-279. https://doi.org/10.1029/95RS03475.

[3] Marzano FS, \& D'Auria G (1998). Model-based prediction of amplitude scintillation variance due to clear-air tropospheric turbulence on Earth-satellite microwave links, IEEE Trans. Antennas Propag. 46 (10), 1506-1518. https://doi.org/10.1109/8.725283.

[4] Vasseur H (1998), Prediction of Tropospheric Scintillation on Satellite Link from Radiosonde. IEEE Ant. Prop., 47, 293-300. https://doi.org/10.1109/8.761069.

[5] Karasawa Y., Yasukawa K. \& Yamada M. (1988). Tropospheric scintillation in the $14 / 11 \mathrm{GHz}$ bands on Earth-space paths with low elevation angles, IEEE Trans. Antennas Propagat. 36 (4), $563-$ 569. https://doi.org/10.1109/8.1146.

[6] Mandeep JS \& Mohamad Tariqul ISLAM (2012). Evaluation of Statistical Tropospheric Scintillation Models Using SUPERBIRDC Satellite for Malaysia. Acta Geophysica, 60 (4), 1180-1191. https://doi.org/10.2478/s11600-012-0024-x.

[7] Dao H, Islam M.R, \& Al-Khateeb K (2013). Rain Fade Slope Model in Satellite Path Based on Data Measured in Heavy Rain Zone, Antennas and Wireless Propagation Letters, IEEE, 12, 50-53. https://doi.org/10.1109/LAWP.2012.2237373.

[8] Pedro Garcia; Jose M.Riera; Ana Benarroch (2002), Propagation Impairment Mitigation for Millimeter wave Radio Systems: Statistics of dry and wet scintillation in Madrid using Intelsat $50 \mathrm{GHz}$ beacon, PM3-013 1st international workshop, COST Action 280.

[9] Mandeep JS (2011). Extracting of tropospheric scintillation propagation data from Ku-band satellite beacon. Int J Phy Sci, 6(11), 2649-2653.

[10] Cheng CY \& Mandeep JS (2014). Comparison of tropospheric scintillation prediction models of the Indonesian climate, Earth, Planets and Space, 66:64. https://doi.org/10.1186/1880-5981-66-64.

[11] Agunlejika, O. Raji TI, \& Adeleke OA (2007). Tropospheric scintillation prediction for some selected cities in Nigeria's tropical climate. Int. Journal of Basic and Applied Science, 9, 10, 12-19.

[12] Vilar E. \& Haddon J. (1984). Measurement and modeling of scintillation intensity to estimate turbulence parameters in an Earth-space path, IEEE Trans.Antenn. Propag. 32, 4, (1984), 340-346. https://doi.org/10.1109/TAP.1984.1143340

[13] Vilar E. \& Haddon J. (1984). Measurement and modeling of scintillation intensity to estimate turbulence parameters in an Earth-space path, IEEE Trans.Antenn. Propag. 32, 4, (1984), 340-346

[14] Van de Kamp MM. Tervonen JK. Salonen E.T \& Pirates Baptista, J.P.V., (1999). Improved models for long-term prediction of tropospheric scintillation on slant paths. IEEE Trans. Antennas. Propagat, 47(2), 249-260. https://doi.org/10.1109/8.761064.

[15] Karasawa Y., Yamada, M. \& Allnutt, JE. A new prediction method for tropospheric scintillation on Earth-Space paths, IEEE Trans. Antennas Propagat., Vol. 36, (Nov. 1988), 1608-1614. https://doi.org/10.1109/8.9712.
[16] Karasawa Y., Yamada, M. \& Allnutt, J. E. A new prediction method for tropospheric scintillation on Earth-Space paths, IEEE Trans. Antennas Propagat., Vol. 36, (Nov. 1988), 1608-1614. https://doi.org/10.1109/8.9712.

[17] Ojo JS \& Falodun SE. (2012). NECOP Propagation Experiment: Rain-rate distributions observations and prediction model comparisons, Int. Journal of Antennas and Propagation, Vol. 2012, https://doi.org/10.1155/2012/913596.

[18] Ojo, JS, Oluwarotimi. EO (2014). Tropical Rainfall Structure Characterization over Two Stations in Southwestern Nigeria for Radiowave Propagation Purposes. Journal of Emerging Trends in Engineering and Applied Sciences, Scholar link Research Institute Journals, 5(2), 116-122.

[19] Garcia-del-Pino, P. Riera JM., \& Benarroch A. (2012). Tropospheric scintillation with concurrent rain attenuation at $50 \mathrm{GHz}$ in $\mathrm{Ma}$ drid. IEEE Trans Antennas and Propagation, Vol. 60 (3), 15781583. https://doi.org/10.1109/TAP.2011.2180326.

[20] Mandeep JS (2011). Anthony Cheng Chen Ye, M.Abdullah, M.Tariqul. Comparison and analysis of tropospheric scintillation models for Northern Malaysia" Acta Astronautica 69, 2-5. https://doi.org/10.1016/i.actaastro.2011.02.014.

[21] Johnson, Norman L.; Kotz, Samuel; Balakrishnan, N. (1994), "14: Lognormal Distributions", Continuous univariate distributions. Vol. 1, Wiley Series in Probability and Mathematical Statistics: Applied Probability and Statistics (2nd ed.), New York: John Wiley \& Sons, ISBN 978-0-471-58495-7, MR 1299979.

[22] Banjo OP \& Vilar E., 1986, "Measurement and modeling of amplitude scintillations on low-elevation earth-space paths and impact on communication systems", IEEE Trans. on Communications Vol. COM-34 pp. 774-780. https://doi.org/10.1109/TCOM.1986.1096617.

[23] Peters G. Marzano FS. D'Auria G \& Riva C., VanhoenackerJanvier D (1997). Evaluation of statistical models for clear-air scintillation prediction using Olympus satellite measurements, Int. J. Satell. Commun. 15, 2, 73-88. https://doi.org/10.1002/(SICI)10991247(199703)15:2<73::AID-SAT570>3.0.CO;2-N 